\title{
Injection of new psychoactive substance snow blow associated with recently acquired HIV infections among homeless people who inject drugs in Dublin, Ireland, 2015
}

C Giese $^{12}$, D Igoe ${ }^{2}$, Z Gibbons ${ }^{3}$, C Hurley ${ }^{4}$, S Stokes ${ }^{3}$, S McNamara ${ }^{3}$, 0 Ennis ${ }^{4}$, K O’Donnell ${ }^{2}$, E Keenan ${ }^{3}$, C De Gascun $^{56}$, F Lyons ${ }^{7}$, M Ward ${ }^{4}$, K Danis ${ }^{18}$, R Glynn ${ }^{4}$, A Waters ${ }^{5}$, M Fitzgerald ${ }^{4}$, on behalf of the outbreak control team ${ }^{9}$

1. European Programme for Intervention Epidemiology Training (EPIET), European Centre for Disease Prevention and Control, (ECDC), Stockholm, Sweden

2. HSE Health Protection Surveillance Centre, Dublin, Ireland

3. HSE National Drug Treatment Centre, Dublin, Ireland

4. Department of Public Health, Dublin, Ireland

5. National Virus Reference Laboratory, University College Dublin, Dublin, Ireland

6. School of Medicine, University College Dublin, Dublin, Ireland

7. St. James's Hospital, Dublin, Ireland

8. French Institute for Public Health Surveillance (Institut de Veille Sanitaire, InVS), Paris, France

9. The members of the team are listed at the end of the article

Correspondence: Coralie Giese (coraliegiese@hotmail.com)

Giese C, Igoe D, Gibbons Z, Hurley C, Stokes S, McNamara S, Ennis O, O’Donnell K, Keenan E, De Gascun C, Lyons F, Ward M, Danis K, Glynn R, Waters A, Fitzgerald $M$. Injection of new psychoactive substance snow blow associated with recently acquired HIV infections among homeless people who inject drugs in Dublin, Ireland, 2015. Euro Surveill. 2015;20(40):pii=30036. DOI: http://dx.doi.org/10.2807/1560-7917.ES.2015.20.40.30036

In February 2015, an outbreak of recently acquired HIV infections among people who inject drugs (PWID) was identified in Dublin, following similar outbreaks in Greece and Romania in 2011. We compared drug and risk behaviours among 15 HIV cases and 39 controls. Injecting a synthetic cathinone, snow blow, was associated with recent HIV infection (AOR: 49; $p=0.003$ ). Prevention and control efforts are underway among PWID in Dublin, but may also be needed elsewhere in Europe.

\section{Background}

In February 2015, the Department of Public Health (DPH), Health Service Executive (HSE) in Dublin, Ireland, identified an unexpected increase in cases of acute HIV infection among people who inject drugs (PWID); three cases were diagnosed p24 antigen-positive in January and February 2015, compared with two cases diagnosed during the whole year in 2014 [1]. Drug treatment clinicians had also identified increased use of a new psychoactive substance (NPS) alphapyrrolidinovalerophenone (a-PVP), known as snow blow, which was being used by 'chaotic' PWID, and which they suspected might be linked to the increase [2]. Clinicians defined the chaotic group as homeless PWID who, if on opioid substitution treatment (OST), required daily attendance at their treatment programme, due to continued use of a variety of other illicit substances in an intensive or chaotic way. We undertook an epidemiological investigation to identify the likely source of this increase.

\section{Epidemiological investigation and case definition}

Clinicians in Dublin provided information on mode of transmission for new HIV diagnoses, and notified new HIV diagnoses in PWID immediately to the DPH. Clinicians asked all cases about the use of snow blow and at-risk sexual practices and whether they were homeless. Cases were crosschecked with data from the Dublin Region Homeless Executive's accommodation client database for periods of accommodation between 1 October 2013 and 30 June 2015.

We defined a case as HIV diagnosis in PWID in Dublin reporting first HIV-positive test since January 2014: a confirmed case had recent HIV infection (one or more of: p24 antigen-positive, history of negative HIV test within 12 months of first positive test, acute seroconversion illness, or recency assay test); a probable case had an HIV diagnosis of unknown duration but with an epidemiological link (sexual intercourse or drug sharing) to a confirmed case. We excluded cases with no evidence of recent infection or epidemiological links to recent HIV diagnoses, and cases previously diagnosed abroad. 


\section{FIGURE 1}

Number of recent HIV infections or diagnoses with epidemiological link to recent infections in PWID in Dublin, by month of first diagnosis, January 2014 to September $2015^{\mathrm{a}}(\mathrm{n}=38)$

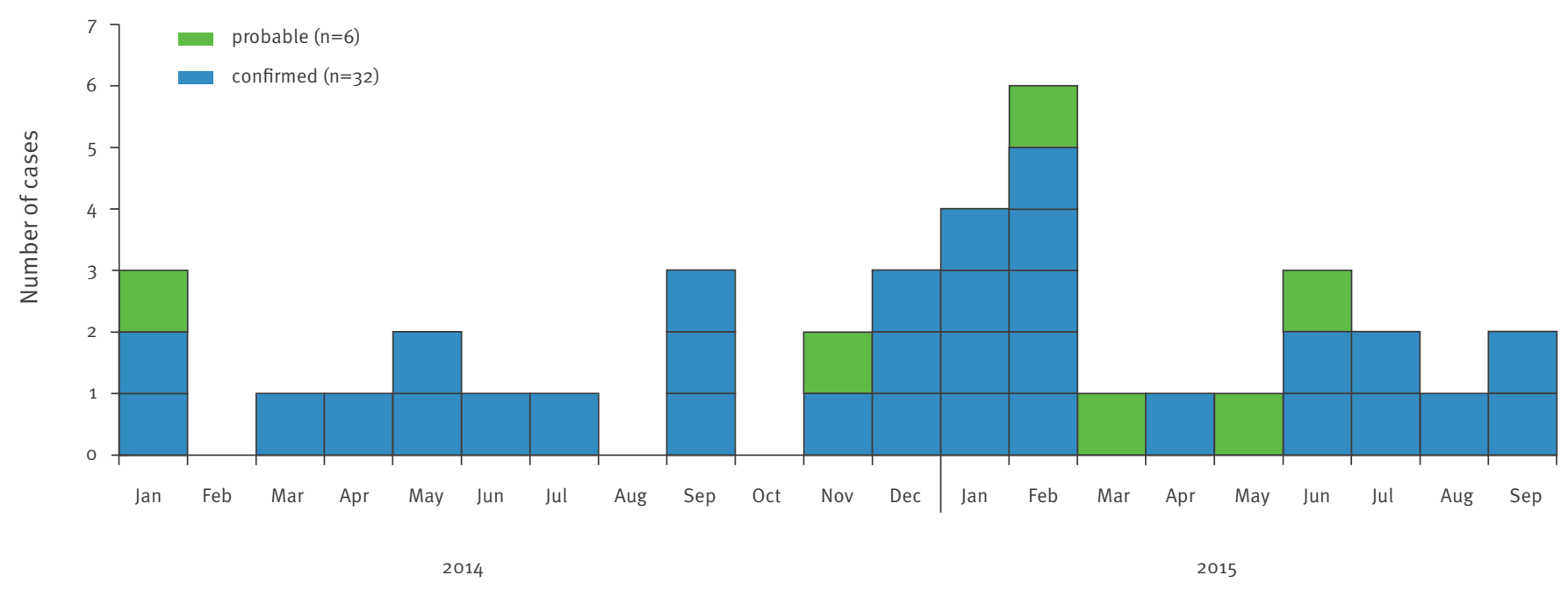

Month of diagnosis

a Data as at 30 September 2015.

\section{TABLE 1}

Distribution of demographic and social characteristics between cases $(n=15)$ and controls $(n=39)$, case-control study, outbreak of recent HIV infection in PWID, Dublin, Ireland, 2015

\begin{tabular}{|c|c|c|c|c|}
\hline \multirow{2}{*}{\multicolumn{2}{|c|}{ Demographic and social characteristics }} & Case & Control & \multirow{2}{*}{ P value } \\
\hline & & $n$ & $n$ & \\
\hline Sex & Female & 6 & 6 & 0.05 \\
\hline \multirow{2}{*}{ Place of residence ${ }^{a}$} & Home/relatives & 7 & 14 & \multirow{2}{*}{0.36} \\
\hline & Homeless/hostels & 7 & 25 & \\
\hline \multirow{2}{*}{\multicolumn{2}{|c|}{ Prison }} & 4 & 0 & 0.001 \\
\hline & & Median (range) & Median (range) & $P$ value \\
\hline \multicolumn{2}{|l|}{ Age (years) } & $35(25-51)$ & $33(19-51)$ & 0.87 \\
\hline \multicolumn{2}{|c|}{ Age at first injection (years) } & $17(11-39)$ & $20(11-44)$ & 0.14 \\
\hline \multicolumn{2}{|c|}{ Duration of injection (years) } & $16(3-26)$ & $11(0-39)$ & 0.14 \\
\hline
\end{tabular}

PWID: people who inject drugs.

a In the previous 12 months.

\section{Case-control study}

We conducted a case-control study among chaotic PWID, injecting within the previous 12 months, residing in Dublin and diagnosed since July 2014. We included confirmed and probable cases. A list of HIV test-negative (within three months) PWID attending the National Drug Treatment Centre (NDTC) in Dublin, registered by NDTC as homeless and requiring daily attendance due to chaotic behaviour, was generated. A random sample was taken from this list, using random figures generated from Excel. Patients on this list who attended and agreed to participate, were included. We conducted face-to-face or telephone interviews, to collect information on drug use including frequency of use, sexual and injecting at-risk practices, and living conditions.
Participants were asked to report exposures for 12 months before HIV positive diagnosis (cases) or negative diagnosis (controls). To examine associations between exposures and infection, we calculated adjusted odds ratios (OR) and 95\% confidence intervals $(\mathrm{Cl})$ using multiple logistic regression. Inclusion criteria used were $p<0.2$ for model entry.

\section{Laboratory investigations}

HIV serological diagnosis was based on the Abbott Architect HIV Ag/Ab Combo, Biomerieux VIDAS HIV Duo Ultra and P24II, and Fujirebio INNO-LIA HIV I/II SCORE assays; recency testing was performed on the Sedia Biosciences HIV-1 LAg-Avidity EIA; and HIV-1 RNA testing was performed on the Abbott Molecular m2000 
TABLE 2

Factors positively associated with recent HIV infection in PWID in univariate analysis, case-control study, outbreak of recent HIV infection in PWID, Dublin, Ireland, 2015

\begin{tabular}{|c|c|c|c|c|c|c|}
\hline \multirow{2}{*}{\multicolumn{2}{|c|}{ Factors positively associated with recent HIV infection }} & \multirow{2}{*}{$\begin{array}{c}\text { Cases } \\
n \\
(\mathrm{~N}=15)\end{array}$} & \multirow{2}{*}{$\begin{array}{c}\text { Controls } \\
\mathrm{n} \\
(\mathrm{N}=39)\end{array}$} & & & \\
\hline & & & & & & \\
\hline Drugs & Administration & & & $\begin{array}{c}\text { Crude } \\
\text { OR }\end{array}$ & $(95 \% \mathrm{Cl})$ & P value \\
\hline Methamphetamine $^{a}$ & Injecting & 7 & 1 & 33 & $(3.2-1535)$ & $<0.001$ \\
\hline Snow blow ${ }^{\mathrm{a}}$ & Injecting & 13 & 7 & 30 & $(4.7-300)$ & $<0.001$ \\
\hline \multirow{2}{*}{ Snow blow (frequency) ${ }^{a}$} & Injecting daily & 8 & $1^{\mathrm{b}}$ & 128 & $(10-1,595)$ & $<0.001$ \\
\hline & Injecting occasionally & 5 & $6^{b}$ & 13 & $(2.1-85)$ & 0.006 \\
\hline Amphetamines $^{c}$ & Using & 3 & 1 & 9.5 & $(0.66-511)$ & 0.03 \\
\hline Other head shop drugs ${ }^{c}$ & Using & 3 & 1 & 9.5 & $(0.66-511)$ & 0.03 \\
\hline Benzodiazepines $^{c}$ & Using & 13 & 23 & 4.5 & $(0.82-46)$ & 0.05 \\
\hline \multirow[t]{2}{*}{ Crack $^{c}$} & Injecting & 1 & 1 & 3.6 & $(0.19-64)$ & 0.39 \\
\hline & Other & 7 & 13 & 1.9 & $(0.55-6.67)$ & 0.3 \\
\hline Heroin $^{c}$ & Injecting & 13 & 38 & 0.17 & $(0.003-3.7)$ & 0.12 \\
\hline \multirow{2}{*}{ Cocaine $^{c}$} & Injecting & 3 & 3 & 3.1 & $(0.54-18)$ & 0.21 \\
\hline & Other & 2 & 5 & 1.2 & $(0.21-7.4)$ & 0.81 \\
\hline \multirow{2}{*}{ Opiates ${ }^{c}$} & Injecting & 0 & 2 & NC & $\mathrm{NC}$ & $\mathrm{NC}$ \\
\hline & Other & 1 & 2 & 1.3 & $(0.10-15)$ & 0.86 \\
\hline \multicolumn{7}{|l|}{ Sexual practices } \\
\hline \multicolumn{2}{|l|}{ Sex with PWID ${ }^{d}$} & 10 & 15 & 3.8 & $(0.87-19)$ & 0.041 \\
\hline \multicolumn{2}{|l|}{ Had sex ${ }^{d}$} & 10 & 25 & 1.3 & $(0.29-6.8)$ & 0.7 \\
\hline \multicolumn{2}{|l|}{ Sex while high ${ }^{\mathrm{d}, \mathrm{e}}$} & 7 & 21 & 0.67 & $(0.08-8.9)$ & 0.68 \\
\hline \multicolumn{2}{|l|}{ Unprotected sex } & 8 & 22 & 0.55 & $(0.05-7.8)$ & 0.54 \\
\hline \multicolumn{7}{|l|}{ At-risk injecting practices } \\
\hline \multicolumn{2}{|c|}{ Use of used needles or syringes } & 8 & 2 & 21 & $(3.1-225)$ & $<0.001$ \\
\hline \multicolumn{2}{|c|}{ Use of used filters } & 6 & 5 & 4.5 & $(0.89-23)$ & 0.026 \\
\hline \multirow{2}{*}{\multicolumn{2}{|c|}{ Use of used containers or spoons for mixing ${ }^{d}$}} & 8 & 10 & 3.7 & $(0.86-16)$ & 0.038 \\
\hline & & Median (range) & Median (range) & & & \\
\hline \multicolumn{2}{|c|}{ Number of different drugs used } & $5(1-9)$ & $3(1-7)$ & $1.7^{f}$ & $(1.1-2.6)$ & 0.011 \\
\hline \multicolumn{2}{|c|}{ Number of people with whom shared or reused works } & $1(0-15)$ & $0(0-10)$ & $1.2^{f}$ & $(0.97-1.5)$ & 0.09 \\
\hline
\end{tabular}

$\mathrm{Cl}$ : confidence interval; NC: Not calculable; OR: odds ratio.

a Reference: not injecting in the previous 12 months or other route of administration.

${ }^{\mathrm{b}}$ Five missing values.

' Reference: not using in the previous 12 months.

${ }^{\mathrm{d}}$ One missing value.

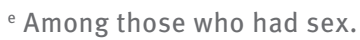

${ }^{\mathrm{f}}$ Per additional unit increase.

RealTime system. HIV subtyping has been described elsewhere [3].

Urine samples of cases attending NDTC were screened by the NDTC laboratory using an in-house Liquid Chromatography/Mass Spectrometry (LC/MS) NPS screening method. Since 2008 , the laboratory of the NDTC has regularly redeveloped its methods in order to meet the challenge of testing for NPS by adding new drugs as they appear in Europe [4,5]. For the purposes of the study, the laboratory of the NDTC developed a tailored NPS LC/MS screening method targeting 26 NPS drugs and common amphetamines in urine. The method covered all relevant drugs detected in the laboratory in recent years including a-PVP which was first added to the NPS screen in 2011.

\section{Results}

\section{Epidemiological investigation and case-control study}

In 2014 and 2015, 38 confirmed and probable cases of HIV subtype B were reported (32 confirmed and six probable) (Figure). Among these, 16 were female; median age was 35 years (range: $24-51$ ). Since January 2014,29 of the 38 had been registered with homeless accommodation services, seven were not registered, and information was not available for two. All females and 13 of the 20 males with information available, 
Factors positively associated with recent HIV infection in multiple regression analysis, case-control study, outbreak of recent HIV infection in PWID, Dublin, Ireland, 2015

\begin{tabular}{|l|c|c|c|}
\hline Factors positively associated with recent HIV infection & $\begin{array}{c}\text { Adjusted } \\
\text { odds ratio }\end{array}$ & 95\% confidence intervals & P value \\
\hline Injecting snow blow & 49 & $3.6-669$ & 0.003 \\
\hline Reusing needles /syringes & 13 & $1.01-177$ & 0.049 \\
\hline Having sex with PWID & 36 & $1.6-782$ & 0.022 \\
\hline Female sex & 3.5 & $0.27-44$ & 0.34 \\
\hline
\end{tabular}

were homeless. In all 18 of the 20 PWID with information available, reported injecting snow blow. At-risk practices, namely sex with PWID, or sex with an HIVpositive partner were reported by 20 of the 38 cases. Thirteen cases reported both using snow blow and sexual at-risk practices. One death was reported.

We recruited 15 cases (12 confirmed, three probable) of 24 cases and 39 controls. Cases were interviewed between 25 May and 18 August 2015 at infectious disease clinics $(n=5)$, drug clinics $(n=7)$, in prison $(n=2)$ or by telephone $(n=1)$. Controls were interviewed at NDTC. All participants but one were enrolled in methadone maintenance treatment.

Cases did not differ from controls in terms of age, age at first injection, duration of injection or living circumstances (Table 1). Females were more likely to become infected than males.

Compared with controls, cases were more likely to have reported injecting methamphetamine, snow blow, consuming amphetamines, other head shop drugs ('legal highs') or benzodiazepines (through various routes of administration) (Table 2).

All cases who reported injecting methamphetamine also reported injecting snow blow. Compared with those who did not inject in the previous 12 months, the odds of recent HIV infection was higher among PWIDs who reported occasional use (weekly, monthly or less than a month) and highest in those who reported injecting snow blow daily (Table 2).

Compared with controls, cases were more likely to have reused needles or syringes, to have used several types of drugs in the previous 12 months and to have had sex with PWID partners.

In the multivariable model, injecting snow blow, using used needles/syringes, and having sex with PWID were the only exposures which remained independently associated with HIV (Table 3 ).

\section{Drug analysis}

Urine samples from 12 cases in the case-control study were tested for NPS. Five were positive: a-PVP $(n=4)$, a-PVP and MDPBP $(n=1)$; seven were negative.

\section{Discussion}

This investigation among homeless chaotic PWID in Dublin is the first evidence of an association between injecting snow blow and recent HIV infection, with daily snow blow injectors being at highest risk. The epidemiological findings are supported by the detection of a-PVP in the urine of cases. Snow blow has been found to contain a-PVP, a second generation cathinone and is closely related to MDPV with similar abuse liability $[2,4,6]$. These findings are consistent with the known stimulant effects of synthetic cathinones and at-risk injecting practices linked to their use [6-11]. Drug treatment clinicians raised concerns that clients who injected snow blow generally exhibited more chaotic behaviours, leading to dis-inhibition, more sharing of needles and syringes, and unprotected sex.

Between 2005 and 2014, more than 81 synthetic cathinone derivatives were reported to the European Union (EU) Early Warning System [12]. Significant quantities of NPS are seized annually in Ireland [13]. In 2011, a-PVP was first detected in urine samples tested for NPS by NDTC. Urines tested for NPS in 2015 contain predominantly a-PVP, pentedrone and MDPBP [5].

Use of synthetic cathinones by homeless chaotic PWID populations has previously been reported in Ireland and in Europe $[6,14]$. In Dublin, there are more than 500 homeless PWID, a significant population at risk of HIV infection [15]. At the time of the study, 252 of ca 520 attendees at the NDTC (48.5\%) were homeless. The general situation regarding homelessness in Dublin has also deteriorated significantly over 2014 and 2015, with a $28 \%$ increase in the number of individuals accessing emergency accommodation in Dublin in the 12 months to June 2015 [16]. The NDTC has a remit for providing OST for homeless PWID, but responding adequately to the needs of these PWID remains challenging.

In Dublin, measures taken include multi-disciplinary work to engage HIV-positive PWID in HIV care and, where appropriate, on antiretroviral therapy: offering HIV testing to PWID; awareness raising among clients, clinicians and networks of PWID about injecting head shop drugs such as snow blow, sexual at-risk behaviours and of the need to engage with methadone and treatment services. Services have been enhanced in terms of contact tracing and active case finding 
and provision of greater access to needle exchange services by strengthening needle/syringe provision through outreach.

There were limitations in undertaking this investigation. Cases and controls were difficult to recruit among homeless chaotic PWID, exposed to illegal and at-risk behaviours. In the descriptive epidemiology, homeless status was determined by asking the patients if they were homeless, and by crosschecking with the homeless service database. If however homeless persons didn't register for any homeless service they might not have been identified as homeless. For the analytical study, controls identified by NDTC as homeless and chaotic were included in the study. However, we found that the homeless status of some participants had changed with a minority no longer reporting homelessness, although all remained chaotic. Cases were more likely to have been in prison. However this was subject to selection bias as we did not select controls in prisons but included cases who were in prison. Behavioural findings were based on self-reported information and some participants were under the effect of drugs while interviewed. This may have had altered the accuracy of measurement of past exposures.

Ireland is the third country reporting an outbreak of HIV among PWID within the past five years in Europe, after Greece and Romania in 2011 [14,17]. A similar increase has also recently been reported in Glasgow and Clyde in June 2015 [18]. Outbreaks of HIV among PWID have also been reported in Israel in 2012 and in Indiana, United States, in early $2015[19,20]$. With low coverage of harm reduction activities such as needle and syringe programmes and OST in most European countries, and a growth of the market in new psychoactive substances, in particular the detection of a -PVP in the 28 EU Member States, the risk of HIV among PWID, and particularly homeless chaotic PWID is likely to be increasing in Europe $[2,12,21]$. It may be of benefit for other countries to consider strengthening prevention programmes for PWID [21-23].

\section{Outbreak Control team members}

Department of Public Health Dublin: Orla Ennis, Margaret Fitzgerald, Caroline Hurley, Ronan Glynn, Mary Ward

National Drug Treatment Services: Zorina Gibbons, Eamonn Keenan, Shay Keating, Seamus Noone

Addiction Services: Des Crowley, Deirdre Dowdall, John Craven

Safety Net Homeless Service: Austin O'Carroll

HSE Social Inclusion: Joe Doyle, Joe Barry

National Virus Reference Laboratory: Jeff Connell, Susie Coughlan, Cillian de Gascun

GUIDE St James's Hospital: Fiona Lyons, Sinead Murphy, Georgina Nagle
Mater Misericordiae University Hospital: Paddy Mallon, Deirdre Morley, Gerard Sheehan

Health Protection Surveillance Centre: Coralie Giese, Derval Igoe, Kate O'Donnell

European Programme for Intervention Epidemiology Training (EPIET), European Centre for Disease Prevention and Control, (ECDC), Stockholm, Sweden: Coralie Giese

\section{Acknowledgements}

The authors wish to thank all those who participated to the study, all interviewers from NDTC, GUIDE clinic, St James's hospital, Curlew road clinic, Dochas centre, the Mater hospital, Thompson Centre, Ms Áine Shine from NDTC Laboratory for testing and analysis of urine samples, Bernie O'Donoghue Hynes Head of Research and Pathie Maphosa Research Assistant from the Dublin Region Homeless Executive. Authors would also like to thank Patricia Garvey EPIET fellow at HPSC for her advice during the analysis, Public Health England for sharing their examples of questionnaire related to drug consumption, and for their Virus Reference Department's (Colindale) assistance with HIV recency testing, and Alicia Barrasa Blanco, EPIET coordinator, for her advice in designing the questionnaire.

\section{Conflict of interest}

None declared.

Authors' contributions

Design of the study: GC, ID, GZ, KE FM, WM, O’DK

Interviews of participants: GZ, GR, LF

Data analysis: GC, ID, DK

Writing of article: GC, ID, DK

Data collection and analysis of cases in outbreak: GC, HC, EO, O’DK

Laboratory diagnosis: DGC, WA

Drug testing: SS, McNS

Article review: GC, ID, GZ, KE, DK, O’DK, SS, McNS, LF, WM, GR, FM, DGC, WA, EO, HC

\section{References}

1. Glynn R, Giese C, Ennis O, Gibbons Z, O’Donnell K, Hurley C, et al. Increase in diagnoses of recently acquired HIV in people who inject drugs. Epi-Insight 2015 Jul;16(7). Available from: http://ndsc.newsweaver.ie/epiinsight/1bumldnml2k?a=1\&p=4 8942718\&t=17517774

2. European Monitoring Centre for Drugs and Drug Addiction (EMCDDA). EMCDDA-Europol Joint Report on a new psychoactive substance: 1-phenyl-2-(1-pyrrolidinyl)-1pentanone (a-PVP). Lisbon: EMCDDA. Sep 2015. Available from http://www.emcdda.europa.eu/publications/joint-reports/ alpha-pvp

3. De GascunCF, WatersA, ReganCM, O’HalloranJ, FarrellG, CoughlanS, et al. Human immunodeficiency virus type 1 in Ireland: phylogenetic evidence for risk group-specific subepidemics. AIDS Res Hum Retroviruses. 2012;28(9):107381. DOI: 10.1089/AID.2011.0301 PMID: 22176216

4. O’ByrnePM, KavanaghPV, McNamaraSM, StokesSM. Screening of stimulants including designer drugs in urine using a liquid chromatography tandem mass spectrometry system.j Anal 
Toxicol. 2013;37(2):64-73. Available from: DOI: 10.1093/jat/ bks091 PMID: 23316030

5. McNamara S, Stokes S, Shine Á, Kilduff R, O’Byrne P. New Psychoactive Substances Prevalence in Samples Tested in the NDTC Laboratory 2010-2015, Poster presented at European Network of Forensic Science Institutes (ENFSI) Drugs Conference 2015 (5th - 8th May 2015) in Dublin Castle, HSE National Drug Treatment Centre, Dublin, Ireland.

6. Van HoutMC, BinghamT. "A costly turn on": patterns of use and perceived consequences of mephedrone based head shop products amongst Irish injectors.Int J Drug Policy. 2012;23(3):188-97. Available from: DOI: 10.1016/j. drugpo.2012.01.008 PMID: 22342322

7. AardeSM, CreehanKM, VandewaterSA, DickersonTJ, TaffeMA. In vivo potency and efficacy of the novel cathinone a-pyrrolidinopentiophenone and 3,4-methylenedioxypyrovalerone: self-administration and locomotor stimulation in male rats.Psychopharmacology (Berl). 2015;232(16):3045-55. Available from: DOI: 10.1007/s00213015-3944-8 PMID: 25925780

8. MarusichJA, AntonazzoKR, WileyJL, BloughBE, PartillaJS BaumannMH. Pharmacology of novel synthetic stimulants structurally related to the "bath salts" constituent 3,4-methylenedioxypyrovalerone (MDPV). Neuropharmacology. 2014;87:206-13. Available from: DOI: 10.1016/j. neuropharm.2014.02.016 PMID: 24594476

9. CameronKN, KolanosR, SolisE, GlennonRA, De FeliceLJ. Bath salts components mephedrone and methylenedioxypyrovalerone (MDPV) act synergistically at the human dopamine transporter.Br J Pharmacol. 2013;168(7):17507. Available from: DOI: 10.1111/bph.12061 PMID: 23170765

10. United Nations Office on Drugs and Crime (UNODC).

FACTSHEET: “New psychoactive substances". VIENNA: UNODC. [Accessed 1 Oct 2015]. Available from: https://www.unodc.org/ documents/scientific/FACTSHEET_NPS.pdf

11. WattersonLR, WattersonE, OliveMF. Abuse liability of novel 'legal high' designer stimulants: evidence from animal models. Behav Pharmacol. 2013;24(5-6):341-55. Available from: DOI: 10.1097/FBP.ob013e3283641ec8 PMID: 23839028

12. European Monitoring Centre for Drugs and Drug Addiction (EMCDDA). Perspective on drugs. Injection of synthetic cathinones. Lisbon: EMCDDA; 2014 May 27. Available from: http://www.emcdda.europa.eu/topics/pods/ synthetic-cathinones-injection

13. An Garda Síochána. Annual Report of An Garda Síochána 2013. Dublin: An Garda Síochána. [Accessed: 1 Oct 2015]. Available from: http://www.garda.ie/Documents/User/Annual\%20 Report\%202013\%20-\%20English.pdf

14. SypsaV, ParaskevisD, MallioriM, NikolopoulosGK, PanopoulosA, KantzanouM, et al. Homelessness and Other Risk Factors for HIV Infection in the Current Outbreak Among Injection Drug Users in Athens, Greece. Am J Public Health. 2015;105(1):196-204. Available from: DOI: 10.2105/ AJPH.2013.301656 PMID: 24524508

15. Barry J. Personal communication, Central Methadone Treatment List.

16. Dublin Region Homeless Executive. Performance Report 2015 relating to the protocol governing delegation of section 10 funding for homeless services to Dublin City Council, Q2 2015. Dublin: Dublin Region Homeless Executive. [Accessed 1 Oct 2015]. Available from: http://www.environ.ie/en/Publications/ DevelopmentandHousing/Housing/FileDownLoad,42531,en.pdf

17. HedrichD, KalamaraE, SfetcuO, PharrisA, NoorA, WiessingL, et al. Human immunodeficiency virus among people who inject drugs: is risk increasing in Europe? Euro Surveill. 2013;18(48):20648. Available from: DOI: 10.2807/1560-7917. ES2013.18.48.20648 PMID: 24308980

18. Scotland HP. (HPS). Weekly Report News. Warning to drug injectors as HIV infections rise. Glasgow: HPS. 30 Jun 2015. Available from: http://www.hps.scot.nhs.uk/bbvsti/wrdetail. aspx? $i d=64359 \&$ wrtype $=2 \#$

19. Katchman E, Savyon M, Chemtob D, Avidor B, Mor O, Wasserman A, et al. An outbreak of primary HIV infection among injecting drug users in Tel Aviv, Israel, associated with changes in illicit drug use practices. 14th European AIDS Conference, Brussels. 2013.

20. Centers for Disease Control and Prevention (CDC),ConradC, BradleyHM, BrozD, BuddhaS, ChapmanEL, GalangRR, et al. . Community Outbreak of HIV Infection Linked to Injection Drug Use of Oxymorphone--Indiana, 2015.MMWR Morb Mortal Wkly Rep. 2015;64(16):443-4.PMID: 25928470

21. European Centre for Disease Prevention and Control (ECDC) / European Monitoring Centre for Drugs and Drug Addiction (EMCDDA). Thematic report: People who inject drugs. Monitoring implementation of the Dublin Declaration on Partnership to Figh HIVH/AIDS in Europe and Central Asia:
2014 progress report. Stockholm: ECDC; Sep 2015. Available from: http://ecdc.europa.eu/en/publications/Publications/ dublin-declaration-people-who-inject-drugs.pdf

22. European Centre for Disease Prevention and Control (ECDC) / European Monitoring Centre for Drugs and Drug Addiction (EMCDDA). Prevention and control of infectious diseases among people who inject drugs. Stockholm: ECDC; Oct 2011. Available from: http://ecdc.europa.eu/en/publications/

Publications/111012_Guidance_ECDC-EMCDDA.pdf

23. European Monitoring Centre for Drugs and Drug Addiction. Drug-related infectious diseases in Europe: update from the EMCDDA expert network. Luxembourg: Publications Office of the European Union. 2015. Available from: http://www.emcdda. europa.eu/attachements.cfm/att_242420_EN_TDo215722ENN. pdf 